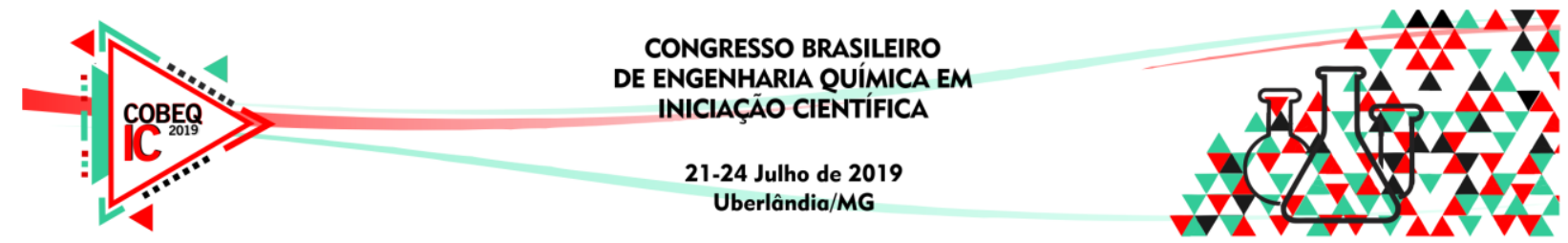

\title{
CINÉTICA DA POLIMERIZAÇÃO QUÍMICA DO PIRROL EM ÁGUA UTILIZANDO CLORETO FÉRRICO COMO OXIDANTE
}

\author{
A. S. BERTAN ${ }^{1}$, A. P. ROMIO ${ }^{1}$, C. Z. BRUSAMARELLO ${ }^{1}$, M. D. DOMENICO ${ }^{1}$ \\ ${ }^{1}$ Universidade Tecnológica Federal do Paraná, Departamento Acadêmico de Engenharia \\ Química \\ E-mail para contato: alessandra.suzin.bertan@gmail.com
}

\begin{abstract}
RESUMO - O objetivo do presente trabalho foi avaliar a conversão experimental e a cinética de polimerização química do pirrol (Pi) em solução aquosa utilizando cloreto férrico como agente oxidante. As reações de polimerização foram realizadas num reator batelada encamisado, com um agitador magnético para manter a agitação constante. As amostras de polipirrol (PPi) foram coletadas do meio reacional ao longo do tempo, sendo filtradas e secas em estufa a $40{ }^{\circ} \mathrm{C}$. Os perfis de conversão gravimétrica foram traçados e os parâmetros cinéticos foram determinados. Os parâmetros de operação utilizados foram: velocidade de agitação (200 e $300 \mathrm{rpm})$, concentração de $\mathrm{Pi}(0,043$ e $0,085 \mathrm{~mol} / \mathrm{L})$, concentração de $\mathrm{FeCl}_{3} .6 \mathrm{H}_{2} \mathrm{O}(0,05,0,1,0,2$ e $2,0 \mathrm{~mol} / \mathrm{L})$ e temperatura $\left(5\right.$ e $\left.20{ }^{\circ} \mathrm{C}\right)$. Por meio deste estudo verificou-se que uma rotação menor é mais eficaz na reação de polimerização. Ainda, o aumento da concentração de ambos os reagentes e também da temperatura promoveu um aumento na conversão, ao longo de todo o experimento. Em relação aos dados de cinética da reação aplicando o método integral, os valores de $\mathrm{R}^{2}$ foram sempre superiores a 0,9 e, bons ajustes foram verificados para ordens de reação do $\mathrm{Pi}$ menores que as do $\mathrm{FeCl}_{3} \cdot 6 \mathrm{H}_{2} \mathrm{O}$. Este resultado é coerente com a literatura $\left(\alpha=1\right.$ para o $\mathrm{Pi}$ e $\beta=2$ para o $\left.\mathrm{FeCl}_{3} .6 \mathrm{H}_{2} \mathrm{O}\right)$ e em acordo com a estequiometria da reação.
\end{abstract}

\section{INTRODUÇÃO}

Surgiu por volta dos anos 50 a ideia de se associar propriedades elétricas dos metais às propriedades mecânicas dos polímeros. Foram incorporadas cargas condutoras aos polímeros (fibras metálicas, negro fumo ou fibras de carbono) produzindo os denominados "polímeros condutores extrínsecos". Recentemente, outra classe de polímeros vem sendo muito estudada, os chamados "polímeros condutores intrínsecos", nos quais a condução de corrente elétrica ocorre sem a incorporação de cargas condutoras (Faez et al., 2000). O polipirrol (abreviatura PPi), polímero estudado neste trabalho, faz parte dessa última classe.

Existem dois métodos comumente empregados na produção do PPi: polimerização eletroquímica e polimerização oxidativa. A polimerização oxidativa é, ainda, muito utilizada por ser um processo rápido que não necessita de equipamentos especiais (KausaiteMinkstimiene et al., 2015). As propriedades dos polímeros dependem de alguns fatores como 


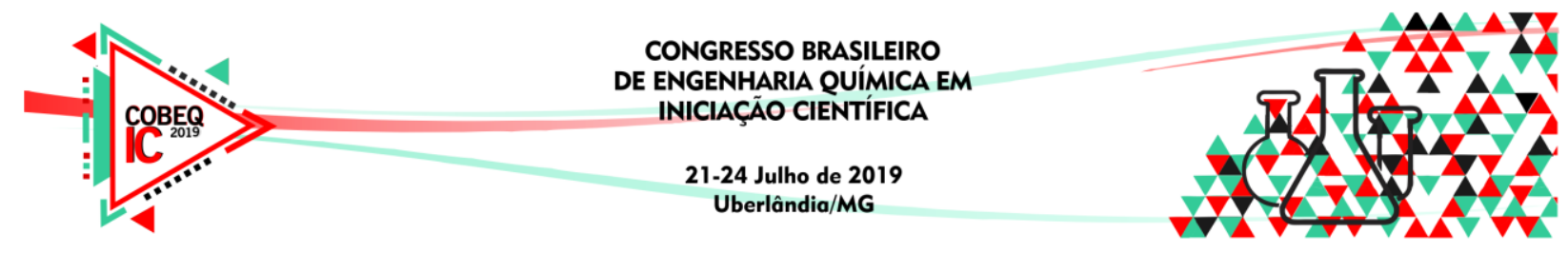

temperatura, tempo de reação, oxidante utilizado, presença de surfactantes e dopantes, entre outros (Omastová et al., 2003; Vilcáková et al., 2004). O agente oxidante, além de iniciar a cadeia de polimerização atua também como dopante (Yaws, 2003; Blinova et al., 2007). A síntese química do polipirrol em solução aquosa, utilizando cloreto férrico como agente oxidante, pode ser representada pela reação dada na Equação 1 (Armes, 1987):

$$
\mathrm{C}_{4} \mathrm{H}_{5} \mathrm{~N}+2,3 \mathrm{FeCl}_{3} \cdot 6 \mathrm{H}_{2} \mathrm{O} \rightarrow 0,25 \mathrm{C}_{4} \mathrm{H}_{3} \mathrm{~N}_{4}^{+} \mathrm{Cl}+2 \mathrm{HCl}+2,3 \mathrm{FeCl}_{2} \cdot 6 \mathrm{H}_{2} \mathrm{O}
$$

Dentro deste contexto, o objetivo do presente trabalho foi avaliar a conversão experimental e a cinética da polimerização química do pirrol em água, usando o cloreto férrico como agente oxidante. Dessa forma, contribuiu-se para o melhor entendimento do processo de síntese química deste polímero, gerando dados fundamentais para a realização de um projeto a nível industrial.

\section{MATERIAIS E MÉTODOS}

\subsection{Reações de Polimerização}

A pesquisa foi realizada nos laboratórios do Curso de Graduação em Engenharia Química da UTFPR, Câmpus Francisco Beltrão, Paraná. Para a síntese química do polipirrol foram utilizados os reagentes: monômero pirrol $\left(\mathrm{C}_{4} \mathrm{H}_{5} \mathrm{~N}\right.$, pureza de $\left.98 \%\right)$ e cloreto de ferro (III) hexahidratado $\left(\mathrm{FeCl}_{3} \cdot 6 \mathrm{H}_{2} \mathrm{O}\right.$, pureza de $\left.97 \%\right)$, ambos da Sigma-Aldrich, gás nitrogênio industrial e água destilada. Inicialmente, o monômero pirrol (abreviatura Pi) foi destilado a vácuo (em evaporador rotativo) e armazenado em frasco âmbar sob atmosfera inerte de $\mathrm{N}_{2} \mathrm{e}$ na ausência de luz, sob refrigeração (Tarifa, 2016).

Na sequência, foram realizadas as reações de polimerização, nas condições operacionais apresentadas na Tabela 1. As variáveis foram selecionadas com base nos trabalhos de Brusamarello (2014) e Tarifa (2016). Um esquema representativo do aparato experimental é mostrado na Figura 1. Os experimentos foram conduzidos dentro da capela de exaustão do laboratório.

Tabela 1 - Condições operacionais dos experimentos de polimerização

\begin{tabular}{cccccccc}
\hline Teste & $\mathbf{T}\left({ }^{\circ} \mathbf{C}\right)$ & $\mathbf{N}(\mathbf{r p m})$ & $\mathbf{V}(\mathbf{m L})$ & $\mathbf{t}(\mathbf{m i n})$ & $\mathbf{C}_{\mathbf{P i}}(\mathbf{m o l} / \mathbf{L})$ & $\mathbf{C}_{\mathbf{F e C 1 3 . 6 H 2 O}}(\mathbf{m o l} / \mathbf{L})$ & Razão Molar \\
\hline 1 & 20 & 200 & 0,05 & 120 & 0,085 & 0,2 & 2,3 \\
2 & 20 & 300 & 0,05 & 120 & 0,085 & 0,2 & 2,3 \\
3 & 20 & 200 & 0,5 & 20 & 0,085 & 0,2 & 2,3 \\
4 & 20 & 200 & 0,5 & 180 & 0,085 & 0,2 & 2,3 \\
\hline 5 & 20 & 200 & 0,5 & 180 & 0,085 & 0,2 & 2,3 \\
6 & 20 & 200 & 0,5 & 180 & 0,043 & 0,1 & 2,3 \\
7 & 20 & 200 & 0,5 & 180 & 0,085 & 2,0 & 23,4 \\
8 & 20 & 200 & 0,5 & 180 & 0,043 & 0,05 & 1,2 \\
\hline 9 & 5 & 200 & 0,5 & 180 & 0,085 & 0,2 & 2,3 \\
\hline
\end{tabular}

${ }^{1}$ Limite inferior do equipamento. 


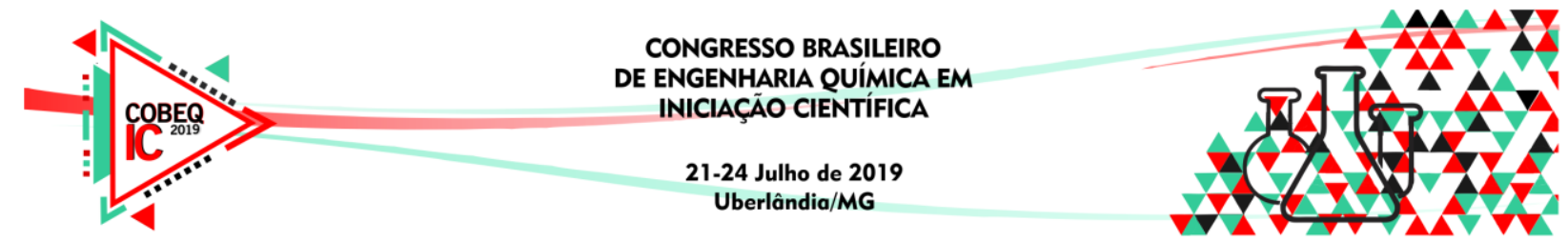

Figura 1 - Esquema geral do aparato experimental. Legenda: (1) bomba submersa, (2) reator encamisado, (3) barra magnética, (4) agitador magnético com aquecimento, (5) capela de exaustão de gases e (6) cuba de alumínio contendo água

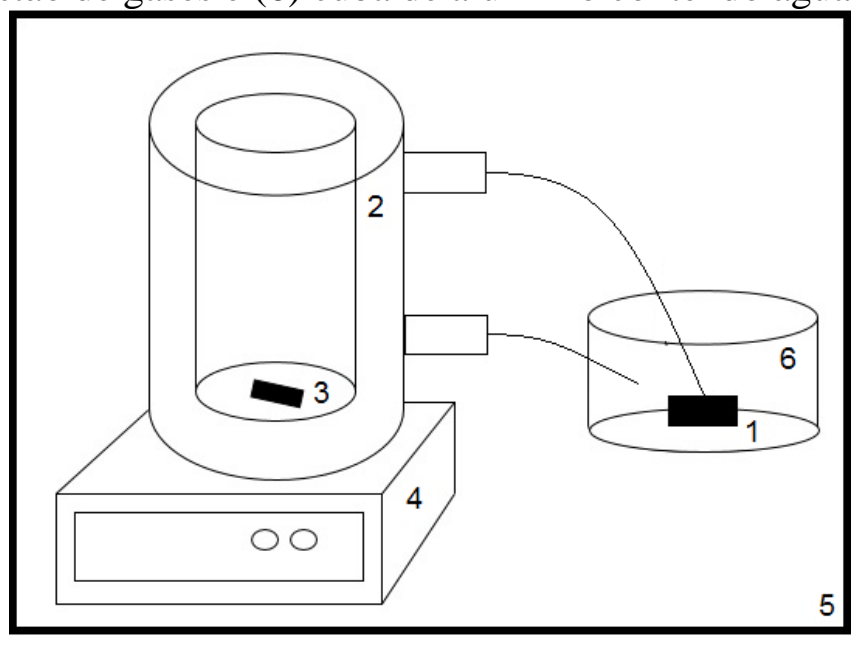

Inicialmente, avaliou-se o efeito da velocidade de agitação e do tempo final de reação na conversão total de Pi em PPi (testes 1 até 4). Na sequência, foram feitos os testes cinéticos com coleta de amostras ao longo do tempo (testes 5 até 9), avaliando-se o efeito da concentração dos reagentes e também da temperatura na polimerização. Os testes 4 e 5 foram feitos nas mesmas condições, porém para o primeiro foi avaliada apenas a conversão total e para o segundo a conversão ao longo do tempo. Os experimentos deste trabalho foram feitos em duplicata, com exceção do teste 5 , que foi realizado em triplicata. O cálculo do erro experimental, por meio dos valores de taxa ou velocidade máxima (dX/dt), indicou um desvio padrão pequeno, de $\pm 0,004 \mathrm{~min}^{-1}$, comprovando o efeito dos parâmetros estudados no resultados de conversão.

O procedimento experimental adotado foi o seguinte: (1) preparação da solução aquosa de $\mathrm{FeCl}_{3}$, (2) agitação constante do meio e aquecimento/resfriamento, de forma a manter o sistema homogêneo, (3) adição do pirrol, gota-a-gota ao reator, sendo este coberto com papel alumínio e, (4) início da contagem do tempo de reação. Ao final, o meio líquido contendo o PPi sólido foi filtrado a vácuo. $\mathrm{O}$ material contido no papel filtro foi lavado várias vezes com água destilada, seco estufa a $40{ }^{\circ} \mathrm{C}$ por $24 \mathrm{~h}$ e, finalmente, pesado em balança analítica. Os testes de cinética foram finalizados somente após a conversão completa.

\subsection{Cálculo e análise dos parâmetros cinéticos}

A análise de dados cinéticos se deu a partir da técnica de aquisição de dados com medições de concentração-tempo em reator batelada (Fogler, 2014). Os dados de massa foram transformados em dados de conversão, e estes, por sua vez, em dados de concentração. A conversão gravimétrica (X) foi calculada pela Equação 2 (Brusamarello et al., 2013; Brusamarello, 2014) na qual $\mathrm{PPi}_{\text {massa }}$ é a massa de PPi retida no filtro (g), $\operatorname{PPr}_{\text {massa }}$ é a massa de PPi removida do meio reacional ( $\mathrm{g}$ ), $\mathrm{Pi}_{\text {add }}$ é a massa de $\mathrm{Pi}$ adicionada ao reator $(\mathrm{g}), \mathrm{M}_{\mathrm{Cl}} \mathrm{e}$ $\mathrm{M}_{\mathrm{Pi}}$ são, respectivamente, a massa molar do cloro e do $\mathrm{Pi}(\mathrm{g} / \mathrm{mol})$ e $\mathrm{MT}_{\text {reator é a massa total }}$ dentro do reator $(\mathrm{g})$. A cinética do presente estudo foi avaliada por meio do método integral e de regressão não linear. Foram obtidos a constante específica da reação de polimerização do 


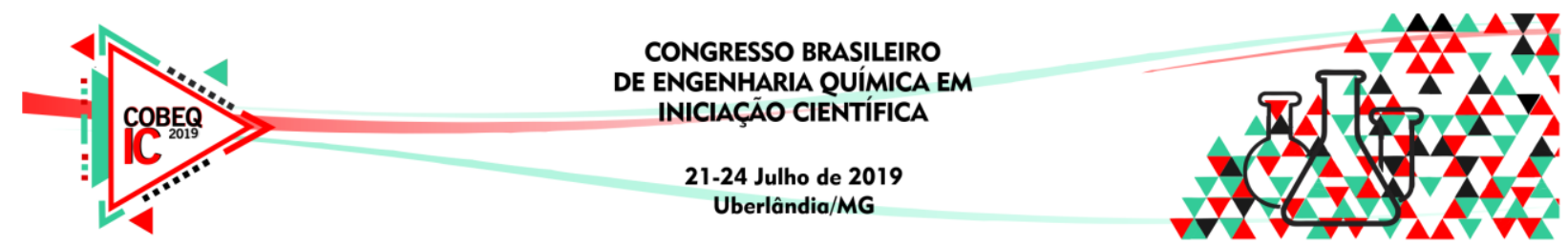

pirrol (k) e a ordem da reação para cada um dos reagentes ( $\alpha$ para o Pi e $\beta$ para o $\mathrm{FeCl}_{3} \cdot 6 \mathrm{H}_{2} \mathrm{O}$ ).

$$
\mathrm{X}=\left[\frac{\mathrm{PPi}_{\text {massa }}}{\left.\frac{\operatorname{PPr}_{\text {massa }} * \mathrm{Pi}_{\text {add }} *\left(1+0.33 *\left(\frac{\mathrm{M}_{\mathrm{Cl}}}{\mathrm{M}_{\mathrm{Pi}}}\right)\right)}{\mathrm{MT}_{\text {reator }}}\right]}\right]
$$

\section{RESULTADOS}

\subsection{Efeito da velocidade de rotação e do tempo na conversão}

Inicialmente, as rotações de $200 \mathrm{rpm}$ e $300 \mathrm{rpm}$ foram testadas para avaliar seu efeito na conversão total (testes 1 e 2). Verificou-se que, num tempo de reação de 120 min e rotação de $200 \mathrm{rpm}$, a conversão atingida foi superior em comparação à de $300 \mathrm{rpm}(0,82$ e 0,76, respectivamente). Isto pode ter ocorrido devido ao efeito de vórtice, impedindo a homogeneidade dos reagentes e conduzindo a uma menor formação de polímero. Além disso, problemas com evaporação do pirrol podem ter ocorrido para a maior velocidade de rotação (Planche et al., 1994). Desta forma, a rotação de $200 \mathrm{rpm}$ foi fixada para os testes seguintes.

Na sequência, avaliou-se o efeito do tempo de reação na conversão total em polímero. Verificou-se que, para tempos reacionais de $20 \mathrm{~min}$ (teste 3), $120 \mathrm{~min}$ (teste 2) e $180 \mathrm{~min}$ (teste 4), a conversão total atingida foi de 0,46, 0,82 e 0,94, respectivamente. Logo, conforme esperado, quanto maior o tempo de reação, maior é a conversão de Pi em PPi. A partir deste resultado, procedeu-se aos testes cinéticos de avaliação da conversão como uma função do tempo, considerando o tempo total de 180 min de reação (teste 5, feito em triplicata). Para a cinética, a conversão final atingida foi superior àquela encontrada no teste de conversão total para 180 min, ultrapassando 100\% de conversão. Para os experimentos de conversão total, pode ter ocorrido a impregnação de polipirrol nas paredes do reator, justificando o menor valor de conversão final encontrado. Por fim, é importante também ressaltar, que a variação do volume de reação 0,05 L para 0,5 L parece não ter afetado a conversão de Pi em PPi.

\subsection{Efeito da concentração dos reagentes e da temperatura no perfil de conversão}

Os perfis de conversão gravimétrica em função do tempo para os testes 5 até 8 são mostrados na Figura 2. Os dados de conversão para estes experimentos, em 90 min de reação, também são apresentados na Tabela 2. Em acordo aos resultados dispostos, ao reduzir as concentrações de ambos os reagentes pela metade (testes 5 e 6), mantendo a razão molar de $\mathrm{FeCl}_{3} .6 \mathrm{H}_{2} \mathrm{O} / \mathrm{Pi}$ de 2,3, ocorre uma diminuição na conversão, ao longo de todo o experimento. Ainda, percebe-se que um aumento de 10 vezes na concentração do oxidante (testes 5 e 7), elevando a razão molar de $\mathrm{FeCl}_{3} .6 \mathrm{H}_{2} \mathrm{O} / \mathrm{Pi}$ para 23,4, eleva de forma brusca a conversão. Logo, ao reduzir a concentração do oxidante pela metade (teste 8), numa razão molar $\mathrm{FeCl}_{3} .6 \mathrm{H}_{2} \mathrm{O} / \mathrm{Pi}$ de 1,2, reduz-se também a conversão de forma substancial. Dessa forma, o agente oxidante utilizado neste trabalho apresentou grande influência na conversão final em polipirrol. 


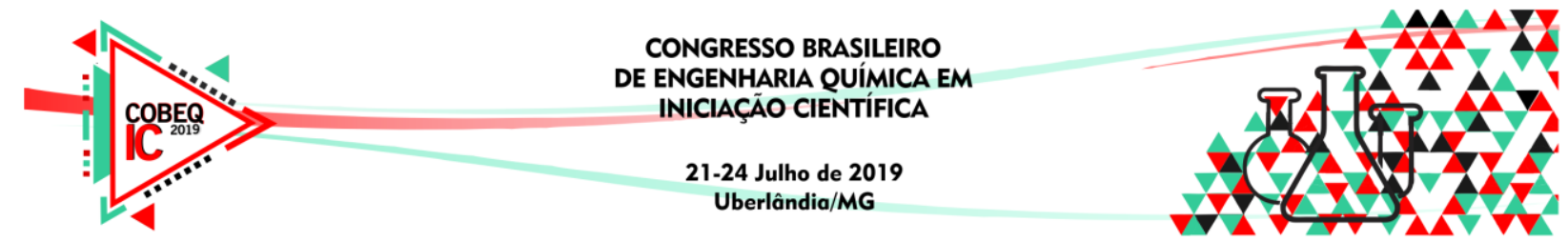

Segundo Planche et al. (1994), a qualidade do polipirrol obtido não é dependente da razão $\mathrm{FeCl}_{3} / \mathrm{Pi}$, mas sim, essencialmente, das concentrações dos dois reagentes. Quanto maiores forem as concentrações, mais rápida será a cinética e melhor será a qualidade do polímero obtido.

Com relação ao efeito da temperatura, verifica-se na Figura 2 que o aumento da temperatura elevou os valores de conversão em todo o perfil considerado. De acordo com a Tabela 2, o aumento de $5{ }^{\circ} \mathrm{C}$ para $20^{\circ} \mathrm{C}$ elevou a conversão de 0,62 para 0,76 em 90 min de reação. A temperatura está diretamente relacionada ao grau de agitação e colisão das moléculas, logo, seu aumento, facilita o avanço da reação.

Figura 2 - Perfis de conversão em função do tempo para os testes de avaliação do efeito da concentração dos reagentes

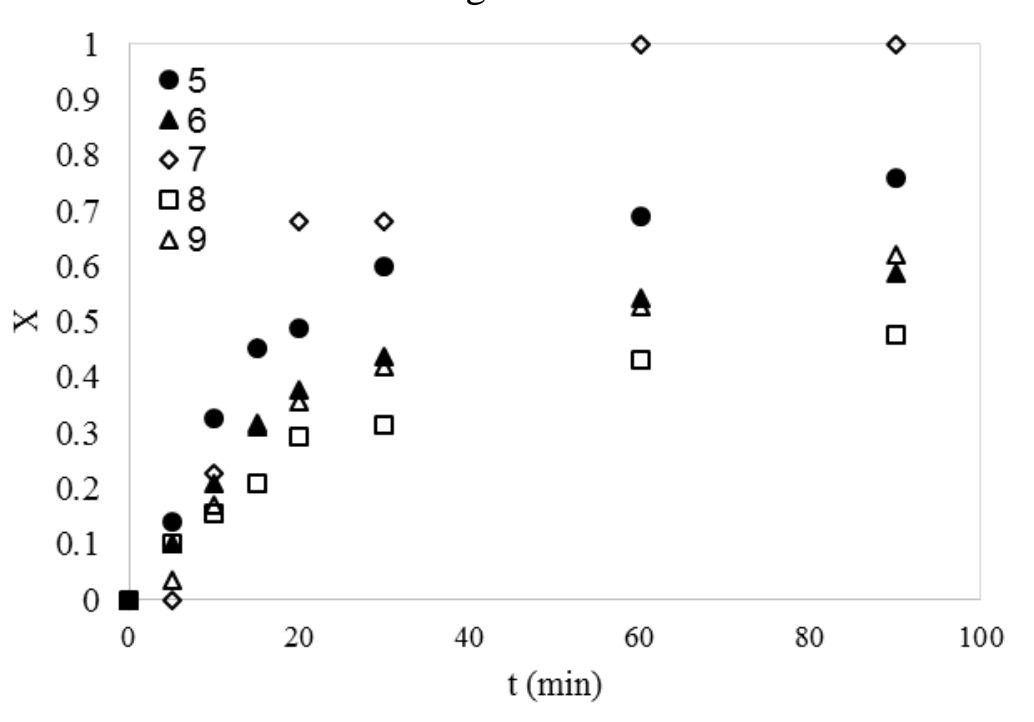

Tabela 2 - Dados de conversão para 90 min de reação

\begin{tabular}{|c|c|c|c|c|c|}
\hline Teste & $\mathbf{T}\left({ }^{\mathbf{0}} \mathbf{C}\right)$ & $\mathbf{C P i}_{\mathbf{P i}}(\mathbf{m o l} / \mathbf{L})$ & $\mathbf{C}_{\mathbf{F e C 1 3 . 6 H 2 O}}(\mathbf{m o l} / \mathbf{L})$ & Razão Molar & $\mathbf{X}_{\mathbf{9 0} \text { min }}$ \\
\hline 5 & 20 & 0,085 & 0,2 & 2,3 & 0,76 \\
\hline 6 & 20 & 0,043 & 0,1 & 2,3 & 0,59 \\
\hline 7 & 20 & 0,085 & 2,0 & 23,4 & 1,00 \\
\hline 8 & 20 & 0,043 & 0,05 & 1,2 & 0,48 \\
\hline 9 & 5 & 0,085 & 0,2 & 2,3 & 0,62 \\
\hline
\end{tabular}

\subsection{Cálculo da ordem de reação e da constante específica da taxa}

Método Integral: Os resultados dos coeficientes de determinação $\left(\mathrm{R}^{2}\right)$ para o ajuste do método integral dos experimentos 5 e 9 são apresentados na Tabela 3. Pode-se verificar que os valores de $\mathrm{R}^{2}$ foram superiores a 0,90 . Ainda, como previsto, os ajustes foram melhores para ordens de reação $\alpha=1$ e $\beta=2$, justificável por meio da estequiometria da reação previamente apresentada pela Equação 1: 2,3 $\mathrm{FeCl}_{3} \cdot 6 \mathrm{H}_{2} 0 / 1 \mathrm{C}_{4} \mathrm{H}_{5} \mathrm{~N}$. 


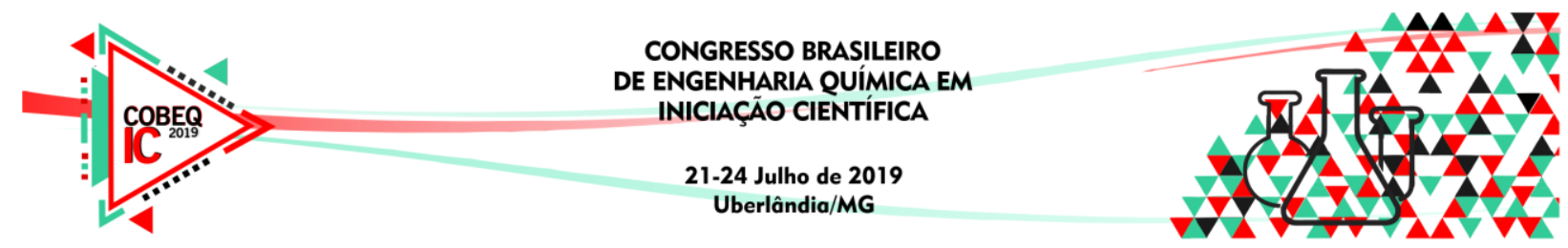

Tabela 3 - Método integral e análise do coeficiente de determinação $\left(\mathrm{R}^{2}\right)$

\begin{tabular}{|c|c|c|c|}
\hline Teste & $\begin{array}{c}\text { Ordem de } \\
\text { reação }(\boldsymbol{n})\end{array}$ & $\left(\mathbf{R}^{\mathbf{2}}\right)$ & $\begin{array}{c}\boldsymbol{k} \\
\left(\mathbf{L}^{\mathbf{2}} \mathbf{\text { min.mol}} \mathbf{2}\right)\end{array}$ \\
\hline \multirow{2}{*}{5} & $\alpha=2 ; \beta=1$ & 0,9593 & 2,21 \\
& $\alpha=1 ; \beta=2$ & 0,9617 & 0,83 \\
\hline \multirow{2}{*}{9} & $\alpha=2 ; \beta=1$ & 0,9042 & 1,10 \\
& $\alpha=1 ; \beta=2$ & 0,9449 & 0,51 \\
\hline
\end{tabular}

\section{REFERENCIAS}

ARMES, S. P. Optimum reaction conditions for the polymerization of pyrrole by iron(III) chloride in aqueous solution. Synthetic Metals., v. 20, p. 365-371, march. 1987.

BLINOVA, N. V.; STEJSKAL, J.; TRCHOVÁ, M.; PROKES, J.; OMASTOVÁ, M. Polyniline and polypyrrole: a comparative study of the preparation. European Plymer Journal., v. 43, p. 2331-3341, 2007.

BRUSAMARELLO, C. Monitoramento de Processos de Polimerização em Meio Disperso. 155f. Tese (Doutorado) - Universidade Federal de Santa Catarina, Florianópolis, 2014.

BRUSAMARELLO, C; SANTOS, L.; AMARAL, M.; BARRA, G.; FORTUNY, M.; SANTOS, A.; ARAUJO, P.; SAYER, C.. Monitoring Pyrrol Polymerization Using On-Line Conductivity Measurements and Neural Networks. Macromolecular Symposia, v. 333, p. 113$121,2013$.

FAEZ, R.; REIS, C.; FREITAS, P. S.; KOSIMA, O. K.; RUGGERI, G.; PAOLI, M-A. D. Polímeros condutores. Química Nova na Escola., v. 11, p. 13-18, 2000.

FAEZ, R.; REZENDE, M. C.; MARTIN, I. M.; PAOLI, M-A. De. Polímeros Condutores Intrínsecos e Seu Potencial em Blindagem de Radiação Eletromagnéticas. Polímeros: Ciência e Tecnologia., v.10, p.130-137, 2000.

FOGLER, H. S. Elementos de Engenharia das Reações Químicas. Rio de Janeiro: Editora LTC, 2014.

KAUSAITE-MINKSTIMIENE, A.; MAZEIKO, V.; REMANAVICIENE, A.; RAMANAVICIUS, A. Evaluation of chemical synthesis of polypyrrole particles. Elsevier., Lithuania., v. 483, p. 224-231, 2015.

OMASTOVÁ, M.; TRCHOVÁ, M.; KORÁVOVÁ, J.; STJSKAL, J. Synthesis and Structural Study of Polypyrroles Prepared in the Presence of Surfactants. Synthetic Metals., Czech Republic, v. 138, p. 447-455, 2013.

TAN, Y.; GHANDI, K. Kinetics and mechanism of pyrrole chemical polymerization. Elsevier., v. 175, p. 183-191, 2013.

TARIFA, Lucas. Síntese química do polipirrol utilizando 1-bromododecano. Francisco Beltrão, PR: Universidade Tecnológica Federal do Paraná, 2016.

VILCÁKOVÁ, J; PALIGOVA, M.; OMASTOVÁ,M.; SÁHA, P.; QUADRAT, O. Swiching Effect in Pressure defformation of Silicon Rubber/ Polypyrrole composites. Synthetic metals., v. 146, p. 121-126, 2004.

YAWS, C. L. Yaws' Handbook of Thermodynamic and Physical Properties of Chemical Compounds. New York: Knovel, 2003. 